\title{
Topological Indices of Dendrimers used in Drug Delivery
}

\author{
T. P. Jude ${ }^{1}$, E. Panchadcharam ${ }^{1 *}$, K. Masilamani ${ }^{2}$ \\ ${ }^{1}$ Department of Mathematics, Faculty of Science, Eastern University, Sri Lanka, Vantharumoolai, \\ Chenkalady 30350, Sri Lanka \\ ${ }^{2}$ Department of Chemistry, Faculty of Science, Eastern University, Sri Lanka, Vantharumoolai, \\ Chenkalady 30350, Sri Lanka
}

Received 7 February 2020, accepted in final revised form 2 June 2020

\begin{abstract}
The topological index is a numerical representation of a molecular structure. In chemical graphs, the atoms and the chemical bonds between them are represented by vertices and edges respectively. Vertex degree based topological indices are the most studied and mostly used type of topological indices. The mostly used vertex degree based topological indices in the field of drug design and developments are the Zagreb index and the Randić index. The structural chemistry of dendrimers could be manipulated by their topological indices to get the specific structure with required properties to deliver the drugs to target carrier vehicle. In this work, topological indices of three types of dendrimers which are used as the drug delivery system were studied and their Zagreb index and the Randić index were calculated using molecular graph theory. Moreover, the other versions of these two indices were also calculated to these dendrimers.
\end{abstract}

Keywords: Topological index; Zagreb index; Randić index; Dendrimers; Drug design, Drug delivery.

(C) 2020 JSR Publications. ISSN: 2070-0237 (Print); 2070-0245 (Online). All rights reserved. doi: http://dx.doi.org/10.3329/jsr.v12i4.45389 J. Sci. Res. 12 (4), 645-655 (2020)

\section{Introduction}

Drug delivery systems have recently received tremendous attention, especially on the targeted drug delivery systems which could transfer drugs into the cytoplasm of targeted cells without harmful toxic effects for healthy tissues or organs [1]. Therefore, development of efficient targeted drug delivery is essential in increasing drug safety and reducing drug-associated toxicity to non-target tissues and organs. Many materials have been demonstrated as drug delivery vehicles traditionally [2,3]. However, many researches are focused on dendrimers in developing the smart drug delivery systems nowadays, since they provide distinctive physiochemical and structural properties compared to other architectural forms of materials that have been used in drug-delivery systems [4-6]. Dendrimers are well-defined globular homogenous three-dimensional structure of nanosize comprising tree-like branches [7,8]. They have narrow

\footnotetext{
Corresponding author: elangop@esn.ac.lk
} 
polydispersity, nanometer size range, which can allow them to easier passage across biological barriers [9]. Therefore, synthesizing the dendrimers with desired properties is challenging task to the pharmaceutical scientists who are involving in developing the drug delivery systems. In this regard, researchers are making attempts to improve physicochemical and biological properties of these agents resulting in increased solubilization, bioavailability, and drug targeting which are crucial in the applications of drug delivery [10]. Hence, architectural chemistry is important to synthesize the dendrimers with the properties which are essential for drug delivery. The topological indices of chemical compounds play a vital role in synthesizing drug delivery system with the key features that support their use as potential drug delivery agents. Therefore, this work focused on the study of topological indices of three types of dendrimers which are used as the drug delivery system. Under this context, this study will pave the path to optimize the architecture of dendrimers with the specific features that are important in using drug deliveries.

The topology of molecules is represented by the topological indices which are the numerical representations of the molecules. They are calculated from the heavy atom graphical depiction of the molecule. Wiener index which was developed in 1947 by the mathematical chemist Wiener [11] was the first topological indices in the study of molecular structure in chemical graph theory or molecular topology. He showed that the Wiener index correlated well with the boiling points of alkanes. Wiener index, Hosoya index or $Z$ index, Zagreb index, Randić index and Balaban $J$ index are the mostly used indices in the field of drug design and development [12-14]. In these indices, the Wiener index, Hosoya index or $Z$ index and the Balaban $J$ indices are non-degree based topological indices, that is, the Wiener index is a distance-based topological index and Balaban $J$ index is a distance sums index. The vertex degree-based topological indices are the mostly studied type of topological indices which play a prominent role in chemical graph theory. Among the above vertex degree-based topological indices, the Zagreb index and the Randić index are the mostly used vertex degree based topological indices in the field of drug design and developments. The Zagreb index and the Randić index were already established for some dendrimers [15-17].

A graph is a structure amounting to a set of objects in which some pairs of the objects are related. The objects are called vertices and each of the related pairs of vertices is called an edge. A graph is denoted by $G=G(V, E)$, where $V=V(G)$ is the nonempty set of vertices and $E=E(G)$ is the set of connected edges if there exists a connection between any pair of vertices in $G$. A connected graph is a graph in which it is possible to get from every vertex in the graph to every other vertex through a series of edges. In a connected graph $G$, the degree of a vertex $v$ in the set of vertices $V(G)$ is defined as the number of vertices of $G$ adjacent to a given vertex $v$ and is denoted by $d_{v}(G)$ or $d_{v}$. Chemical graph theory is a branch of mathematics which combines graph theory and chemistry. In chemical graph theory, a chemical graph or molecular graph is a representation of the structural formula of a chemical compound in terms of graph theory. In a chemical graph, the vertices represent atoms and the edges represent bonds between 
those atoms of any chemical structures. It is noted that the degree of any vertex in a chemical graph is at most four. The concept of degree is closely related to the concept of valence bond in chemistry.

The Zagreb index which was introduced by Gutman and Trinajstić [18] during the analysis of the structure-dependency of total-electron energy is one of the oldest degreebased topological indices. The Zagreb index has different versions; the first Zagreb index $M_{1}(G)$ was defined as

$$
\mathrm{M}_{1}(\mathrm{G})=\sum_{\mathrm{v} \in \mathrm{V}(\mathrm{G})}\left(\mathrm{d}_{\mathrm{v}}\right)^{2}=\sum_{\mathrm{uv} \in \mathrm{E}(\mathrm{G})}\left(\mathrm{d}_{\mathrm{u}}+\mathrm{d}_{\mathrm{v}}\right)
$$

and the second Zagreb index $M_{2}(G)$ was defined as

$$
M_{2}(G)=\sum_{u v \in E(G)} d_{u} d_{v}
$$

The second modified Zagreb index which was introduced by Hao [19] and was defined as

$$
{ }^{m} M_{2}(G)=\sum_{u v \in E(G)} \frac{1}{d_{u} d_{v}} .
$$

The Randić index which is also one of the oldest topological indices was introduced by Randić [20]. This was defined as

$$
R(G)=\sum_{u v \in E(G)} \frac{1}{\sqrt{d_{u} d_{v}}}
$$

The Randić index is one of the most studied, most often applied and most popular degree-based topological index in the field of drug design. This index was globalized and recognized as the generalized Randić index and was defined as

$$
\mathrm{R}_{\alpha}(\mathrm{G})=\sum_{\mathrm{uv} \in \mathrm{E}(\mathrm{G})}\left(\mathrm{d}_{\mathrm{u}} \mathrm{d}_{\mathrm{v}}\right)^{\alpha},
$$

where $\alpha$ is an arbitrary real number [21]. When $\alpha=-\frac{1}{2}$, the above generalized Randić index becomes the (original) Randić index.

The reciprocal Randić index was defined as

$$
R R(G)=\sum_{u v \in E(G)} \sqrt{d_{u} d_{v}}
$$

This is actually a special case of the Randić index when $\alpha=\frac{1}{2}$. This index was first encountered in a paper by Favaron et al. [22].

The reduced second Zagreb index was defined as

$$
\mathrm{RM}_{2}(\mathrm{G})=\sum_{\mathrm{uv} \in \mathrm{E}(\mathrm{G})}\left(\mathrm{d}_{\mathrm{u}}-1\right)\left(\mathrm{d}_{\mathrm{v}}-1\right)
$$

and the reduced reciprocal Randić index was defined as

$$
\operatorname{RRR}(G)=\sum_{u v \in E(G)} \sqrt{\left(d_{u}-1\right)\left(d_{v}-1\right)}
$$

The reciprocal Randić index, the reduced second Zagreb index and the reduced second Zagreb index actually appeared in the literature earlier but did not given much attention by the mathematical chemists. Gutman et al., [23] presented the main mathematical properties and established the correlating abilities with respect to characteristic physico- 
chemical properties of alkanes. Because of these presentations, these indices become popular among the vertex degree based topological indices nowadays.

\section{Calculation and Results}

In this paper, we considered three biodegradable dendrimers: polyacetal dendrimers with polyhedral oligomeric silsesquioxane (POSS) core and a polyester dendrimer.

First, we considered the polyacetal dendrimer with polyhedral oligomeric silsesquioxane (POSS) core. By observing the structure as it shows in Fig. 1, we inferred five partitions of the edge set which are:

$$
\begin{aligned}
& E_{1}(G)=\left\{e=u v \in E(G): d_{u}=1 \text { and } d_{v}=2\right\}, \\
& E_{2}(G)=\left\{e=u v \in E(G): d_{u}=1 \text { and } d_{v}=3\right\}, \\
& E_{3}(G)=\left\{e=u v \in E(G): d_{u}=d_{v}=2\right\}, \\
& E_{4}(G)=\left\{e=u v \in E(G): d_{u}=2 \text { and } d_{v}=3\right\}, \\
& E_{5}(G)=\left\{e=u v \in E(G): d_{u}=2 \text { and } d_{v}=4\right\} .
\end{aligned}
$$

The number of edges of the edge set $E_{1}(G)$ are given by $\left|E_{1}(G)\right|=16,32,64$ and $8 \times 2^{n}$ for the $1^{\text {st }}, 2^{\text {nd }}, 3^{\text {rd }}$ and the $n^{\text {th }}$ generations respectively. Similarly, the number of edges of the other edge sets are given by $\left|E_{2}(G)\right|=32,96,224$ and $32 \times 2^{n}-$ $32 ;\left|E_{3}(G)\right|=128,384,896$ and $128 \times 2^{n}-128$; and $\left|E_{4}(G)\right|=84,264,616$ and $88 \times 2^{n}-88$ for the $1^{\text {st }}, 2^{\text {nd }}, 3^{\text {rd }}$ and the $n^{\text {th }}$ generations. Further, $\left|E_{5}(G)\right|=32$ for all the generations. Here $n$ is the number of growth of the dendrimers.

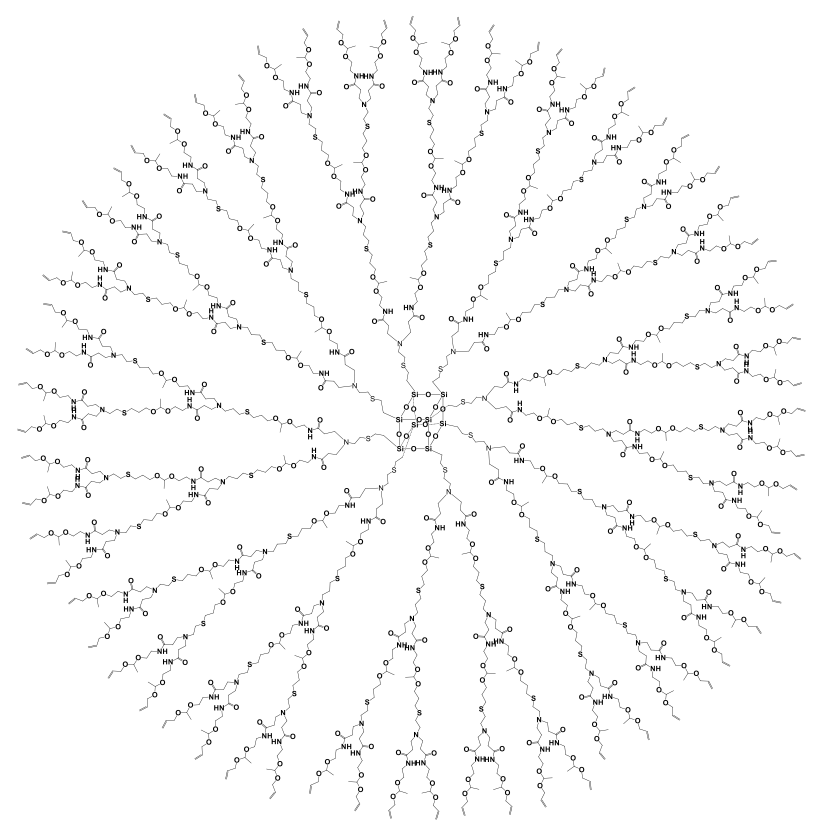

Fig 1. Structure of polyacetal dendrimer with a polyhedral oligomeric silsesquioxane (POSS) core [24]. 


\subsection{Theorem 1}

Let $\mathrm{G}$ be the Schematic representation of third-generation polyacetal dendrimer [24]. Then the first Zagreb index $M_{1}(G)$, the second Zagreb index $M_{2}(G)$, the second modified Zagreb index ${ }^{m} M_{2}(G)$ and the reduced second Zagreb index $R M_{2}(G)$ for $\mathrm{G}$ are

1. $M_{1}(G)=1104 \times 2^{n}-1272$.

2. $M_{2}(G)=1152 \times 2^{n}-1392$.

3. ${ }^{m} M_{2}(G)=\frac{184}{3} \times 2^{n}-\frac{184}{3}$.

4. $R M_{2}(G)=304 \times 2^{n}-400$.

\section{Proof}

Using the edge partitions of the schematic representation of polyacetal dendrimer and by the formulas of different version of the Zagreb indices, we get

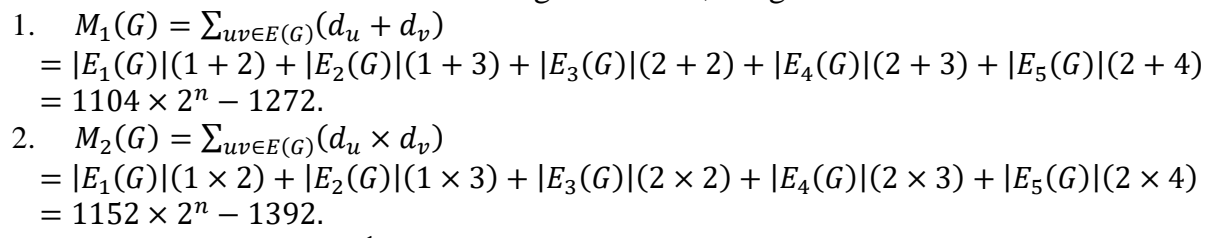

3. ${ }^{m} M_{2}(G)=\sum_{u v \in E(G)} \frac{1}{d_{u} d_{v}}$ $=\left|E_{1}(G)\right| \frac{1}{1 \times 2}+\left|E_{2}(G)\right| \frac{1}{1 \times 3}+\left|E_{3}(G)\right| \frac{1}{2 \times 2}+\left|E_{4}(G)\right| \frac{1}{2 \times 3}+\left|E_{5}(G)\right| \frac{1}{2 \times 4}$ $=\frac{184}{3} \times 2^{n}-\frac{184}{3}$.

4. $\quad R M_{2}(G)=\sum_{u v \in E(G)}\left(d_{u}-1\right)\left(d_{v}-1\right)$ $=\left|E_{1}(G)\right|(1-1)(2-1)+\left|E_{2}(G)\right|(1-1)(3-1)+\left|E_{3}(G)\right|(2-1)(2-1)$ $=\left(304 \times 2^{n}-400\right)$

\subsection{Theorem 2}

Let $G$ be the schematic representation of third-generation polyacetal dendrimer [24]. Then the Randić index $R(G)$, the reciprocal Randić index $R R(G)$ and the reduced reciprocal Randić index $R R R(G)$ for $G$ are

\section{Proof.}

1. $R(G)=\left(\frac{8}{\sqrt{2}}+\frac{32}{\sqrt{3}}+\frac{88}{\sqrt{6}}+64\right) \times 2^{n}-\left(\frac{16}{\sqrt{2}}+\frac{32}{\sqrt{3}}+\frac{88}{\sqrt{6}}+64\right)$.

2. $R R(G)=(8 \sqrt{2}+32 \sqrt{3}+88 \sqrt{6}+256) 2^{n}-(64 \sqrt{2}+32 \sqrt{3}+88 \sqrt{6}+256)$.

3. $R R R(G)=(128+88 \sqrt{2}) 2^{n}-(128+88 \sqrt{2}+32 \sqrt{3})$.

Using the edge partitions of the polyacetal dendrimer and by the formulas of different version of the Randić indices, we get

1. $R(G)=\sum_{u v \in E(G)} \frac{1}{\sqrt{d_{u} d_{v}}}$

$$
\begin{aligned}
& =\left|E_{1}(G)\right| \frac{1}{\sqrt{1 \times 2}}+\left|E_{2}(G)\right| \frac{1}{\sqrt{1 \times 3}}+\left|E_{3}(G)\right| \frac{1}{\sqrt{2 \times 2}}+\left|E_{4}(G)\right| \frac{1}{\sqrt{2 \times 3}}+\left|E_{5}(G)\right| \frac{1}{\sqrt{2 \times 4}} \\
& =\left(\frac{8}{\sqrt{2}}+\frac{32}{\sqrt{3}}+\frac{88}{\sqrt{6}}+64\right) \times 2^{n}-\left(\frac{16}{\sqrt{2}}+\frac{32}{\sqrt{3}}+\frac{88}{\sqrt{6}}+64\right) .
\end{aligned}
$$

2. $R R(G)=\sum_{u v \in E(G)} \sqrt{d_{u} d_{v}}$ 


$$
\begin{aligned}
&=\left|E_{1}(G)\right| \sqrt{1 \times 2}+\left|E_{2}(G)\right| \sqrt{1 \times 3}+\left|E_{3}(G)\right| \sqrt{2 \times 2}+\left|E_{4}(G)\right| \sqrt{2 \times 3}+\left|E_{5}(G)\right| \sqrt{2 \times 4} \\
&=(8 \sqrt{2}+32 \sqrt{3}+88 \sqrt{6}+256) 2^{n}-(64 \sqrt{2}+32 \sqrt{3}+88 \sqrt{6}+256) . \\
& \text { 3. } \quad R R R(G)=\sum_{u v \in E(G)} \sqrt{\left(d_{u}-1\right)\left(d_{v}-1\right)} \\
&=\left|E_{1}(G)\right| \sqrt{(1-1)(2-1)}+\left|E_{2}(G)\right| \sqrt{(1-1)(3-1)}+\left|E_{3}(G)\right| \sqrt{(2-1)(2-1)} \\
& \quad+\left|E_{4}(G)\right| \sqrt{(2-1)(3-1)}+\left|E_{5}(G)\right| \sqrt{(2-1)(4-1)} \\
&=(128+88 \sqrt{2}) 2^{n}-(128+88 \sqrt{2}+32 \sqrt{3}) .
\end{aligned}
$$

Now we consider the polyester dendrimer from sequential click coupling of asymmetrical monomers. By observing the structure as shows in Fig. 2, we inferred three partitions of the edge set which are:

$E_{1}(G)=\left\{e=u v \in E(G): d_{u}=1\right.$ and $\left.d_{v}=2\right\}$,

$E_{2}(G)=\left\{e=u v \in E(G): d_{u}=1\right.$ and $\left.d_{v}=3\right\}$,

$E_{3}(G)=\left\{e=u v \in E(G): d_{u}=d_{v}=2\right\}$,

$E_{4}(G)=\left\{e=u v \in E(G): d_{u}=2\right.$ and $\left.d_{v}=3\right\}$,

$E_{5}(G)=\left\{e=u v \in E(G): d_{u}=d_{v}=3\right\}$.

The number of edges of the edge set $E_{1}(G)$ are given by $\left|E_{1}(G)\right|=4,8,16$ and $2^{n+1}$ for the $1^{\text {st }}, 2^{\text {nd }}, 3^{\text {rd }}$ and the $n^{\text {th }}$ generations respectively. Similarly, the number of edges of the other edge sets are given by $\left|E_{2}(G)\right|=12,36,84$ and $12 \times 2^{n}-12 ;\left|E_{3}(G)\right|=31,87$, 199 and $28 \times 2^{n}-25 ;\left|E_{4}(G)\right|=22,66,184$ and $22 \times 2^{n}-22$ and $\left|E_{5}(G)\right|=4,12,28$ and $4 \times 2^{n}-4$ for the $1^{\text {st }}, 2^{\text {nd }}, 3^{\text {rd }}$ and the $n^{\text {th }}$ generations. Here $n$ is the number of growth of the dendrimers.

\subsection{Theorem 3}

Let $G$ be thethird-generation polyester dendrimer from sequential click coupling of asymmetrical monomers [24]. Then the first Zagreb index $M_{1}(G)$, the second Zagreb index $M_{2}(G)$, the second modified Zagreb index ${ }^{m} M_{2}(G)$ and the reduced second Zagreb index $R M_{2}(G)$ for $\mathrm{G}$ are

1. $M_{1}(G)=300 \times 2^{n}-282$.

2. $M_{2}(G)=320 \times 2^{n}-304$.

3. ${ }^{m} M_{2}(G)=\frac{145}{9} \times 2^{n}-\frac{517}{36}$.

4. $R M_{2}(G)=88 \times 2^{n}-85$.

\section{Proof.}

Using the edge partitions of the polyester dendrimer from sequential click coupling of asymmetrical monomers and by the formulas of different version of the Zagreb indices, we get

$$
\text { 1. } \begin{aligned}
& M_{1}(G)=\sum_{u v \in E(G)}\left(d_{u}+d_{v}\right) \\
= & \left|E_{1}(G)\right|(1+2)+\left|E_{2}(G)\right|(1+3)+\left|E_{3}(G)\right|(2+2)+\left|E_{4}(G)\right|(2+3)+\left|E_{5}(G)\right|(3+3) \\
= & 300 \times 2^{n}-282 . \\
\text { 2. } & M_{2}(G)=\sum_{u v \in E(G)}\left(d_{u} \times d_{v}\right) \\
= & \left|E_{1}(G)\right|(1 \times 2)+\left|E_{2}(G)\right|(1 \times 3)+\left|E_{3}(G)\right|(2 \times 2)+\left|E_{4}(G)\right|(2 \times 3)+\left|E_{5}(G)\right|(3 \times 3) \\
= & 320 \times 2^{n}-304 .
\end{aligned}
$$

3. ${ }^{m} M_{2}(G)=\sum_{u v \in E(G)} \frac{1}{d_{u} d_{v}}$

$$
=\left|E_{1}(G)\right| \frac{1}{1 \times 2}+\left|E_{2}(G)\right| \frac{1}{1 \times 3}+\left|E_{3}(G)\right| \frac{1}{2 \times 2}+\left|E_{4}(G)\right| \frac{1}{2 \times 3}+\left|E_{5}(G)\right| \frac{1}{3 \times 3}
$$




$$
=\frac{145}{9} \times 2^{n}-\frac{517}{36} .
$$

4. $\quad R M_{2}(G)=\sum_{u v \in E(G)}\left(d_{u}-1\right)\left(d_{v}-1\right)$

$=\left|E_{1}(G)\right|(1-1)(2-1)+\left|E_{2}(G)\right|(1-1)(3-1)+\left|E_{3}(G)\right|(2-1)(2-1)$

$=\left(88 \times 2^{n}-85\right)$.

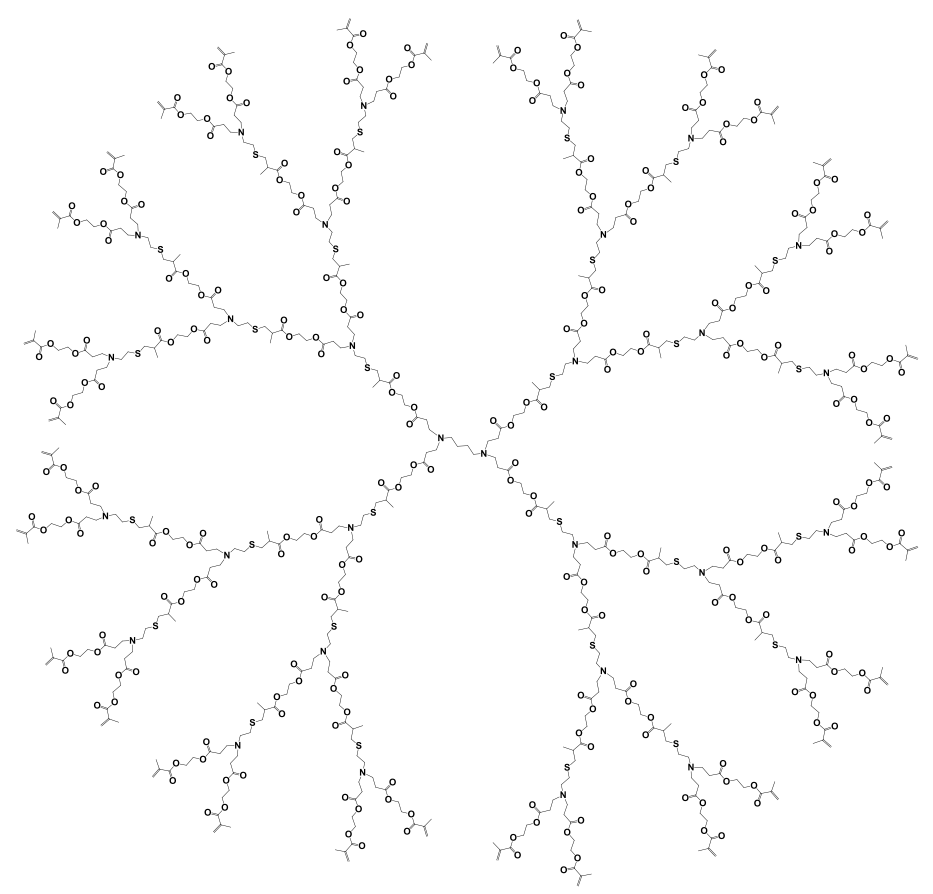

Fig. 2. Structure of polyester dendrimer [24].

\subsection{Theorem 4}

Let $\mathrm{G}$ be the third-generation polyester dendrimer from sequential click coupling of asymmetrical monomers [24]. Then the Randić index $R(G)$, the reciprocal Randić index $R R(G)$ and the reduced reciprocal Randić index $R R R(G)$ for $G$ are

1. $R(G)=\left(\sqrt{2}+\frac{12}{\sqrt{3}}+\frac{22}{\sqrt{6}}+\frac{46}{3}\right) \times 2^{n}-\left(\frac{12}{\sqrt{3}}+\frac{22}{\sqrt{6}}+\frac{83}{6}\right)$.

2. $R R(G)=(2 \sqrt{2}+12 \sqrt{3}+22 \sqrt{6}+68) 2^{n}-(12 \sqrt{3}+22 \sqrt{6}+62)$.

3. $\operatorname{RRR}(G)=(36+22 \sqrt{2}) 2^{n}-(33+22 \sqrt{2})$.

Proof:

Using the edge partitions of the structure and by the formulas of different version of the Randić indices, we get

1. $R(G)=\sum_{u v \in E(G)} \frac{1}{\sqrt{d_{u} d_{v}}}$ $=\left|E_{1}(G)\right| \frac{1}{\sqrt{1 \times 2}}+\left|E_{2}(G)\right| \frac{1}{\sqrt{1 \times 3}}+\left|E_{3}(G)\right| \frac{1}{\sqrt{2 \times 2}}+\left|E_{4}(G)\right| \frac{1}{\sqrt{2 \times 3}}+\left|E_{5}(G)\right| \frac{1}{\sqrt{3 \times 3}}$ 


$$
=\left(\sqrt{2}+\frac{12}{\sqrt{3}}+\frac{22}{\sqrt{6}}+\frac{46}{3}\right) \times 2^{n}-\left(\frac{12}{\sqrt{3}}+\frac{22}{\sqrt{6}}+\frac{83}{6}\right) .
$$

2. $R R(G)=\sum_{u v \in E(G)} \sqrt{d_{u} d_{v}}$

$$
\begin{gathered}
=\left|E_{1}(G)\right| \sqrt{1 \times 2}+\left|E_{2}(G)\right| \sqrt{1 \times 3}+\left|E_{3}(G)\right| \sqrt{2 \times 2}+\left|E_{4}(G)\right| \sqrt{2 \times 3}+\left|E_{5}(G)\right| \sqrt{3 \times 3} \\
=(2 \sqrt{2}+12 \sqrt{3}+22 \sqrt{6}+68) 2^{n}-(12 \sqrt{3}+22 \sqrt{6}+62) .
\end{gathered}
$$

3. $\operatorname{RRR}(G)=\sum_{u v \in E(G)} \sqrt{\left(d_{u}-1\right)\left(d_{v}-1\right)}$

$$
\begin{aligned}
& =\left|E_{1}(G)\right| \sqrt{(1-1)(2-1)}+\left|E_{2}(G)\right| \sqrt{(1-1)(3-1)}+\left|E_{3}(G)\right| \sqrt{(2-1)(2-1)} \\
& \quad+\left|E_{4}(G)\right| \sqrt{(2-1)(3-1)+\left|E_{5}(G)\right| \sqrt{(3-1)(3-1)}} \\
& =(36+22 \sqrt{2}) 2^{n}-(33+22 \sqrt{2}) .
\end{aligned}
$$

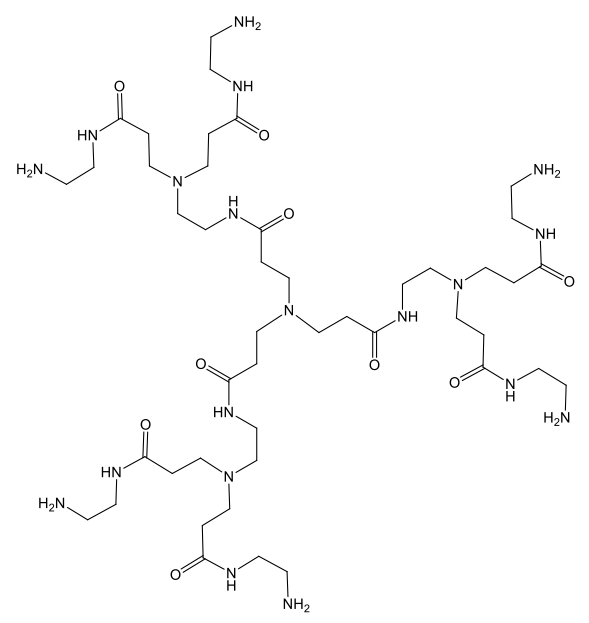

Fig. 3. Structure of polyamidoamine (PAMAM) dendrimer [25].

Finally, we consider the polyamidoamine (PAMAM) dendrimer [25]. By observing the structure as it shows in Fig. 3, we inferred four partitions of the edge set which are:

$E_{1}(G)=\left\{e=u v \in E(G): d_{u}=1\right.$ and $\left.d_{v}=2\right\}$,

$E_{2}(G)=\left\{e=u v \in E(G): d_{u}=1\right.$ and $\left.d_{v}=3\right\}$,

$E_{3}(G)=\left\{e=u v \in E(G): d_{u}=d_{v}=2\right\}$,

$E_{4}(G)=\left\{e=u v \in E(G): d_{u}=2\right.$ and $\left.d_{v}=3\right\}$.

The number of edges of the edge set $E_{1}(G)$ are given by $\left|E_{1}(G)\right|=6,24,96$ and $\frac{3}{2} \times 2^{2 n}$ for the $1^{\text {st }}, 2^{\text {nd }}, 3^{\text {rd }}$ and the $n^{\text {th }}$ generations respectively. Similarly, the number of edges of the other edge sets are given by $\left|E_{2}(G)\right|=9,45,189$ and $3 \times 2^{2 n}-3$; $\left|E_{3}(G)\right|=27,135,567$ and $9 \times 2^{2 n}-9$; and $\left|E_{4}(G)\right|=30,138,570$ and $9 \times 2^{2 n}-6$ for the $1^{\text {st }}, 2^{\text {nd }}, 3^{\text {rd }}$ and the $n^{\text {th }}$ generations. Here $n$ is the number of growth of the dendrimers.

\subsection{Theorem 5}

Let $G$ be the Schematic representation of the second-generation poly (amidoamine) dendrimer. Then the first Zagreb index $M_{1}(G)$, the second Zagreb index $M_{2}(G)$, the 
second modified Zagreb index ${ }^{\mathrm{m}} M_{2}(G)$ and the reduced second Zagreb index $R M_{2}(G)$ for $G$ are

1. $M_{1}(G)=195 \times 2^{2 n-1}-78$.

2. $M_{2}(G)=102 \times 2^{2 n-1}-81$.

3. ${ }^{m} M_{2}(G)=11 \times 2^{2 n-1}-\frac{17}{4}$.

4. $R M_{2}(G)=27 \times 2^{2 n}-11$.

\section{Proof.}

Using the edge partitions of the second-generation poly (amidoamine) dendrimer and by the formulas of different versions of the Zagreb indices, we get

1. $M_{1}(G)=\sum_{u v \in E(G)}\left(d_{u}+d_{v}\right)$

$=\left|E_{1}(G)\right|(1+2)+\left|E_{2}(G)\right|(1+3)+\left|E_{3}(G)\right|(2+2)+\left|E_{4}(G)\right|(2+3)$

$=195 \times 2^{2 n-1}-78$.

2. $M_{2}(G)=\sum_{u v \in E(G)}\left(d_{u} \times d_{v}\right)$

$=\left|E_{1}(G)\right|(1 \times 2)+\left|E_{2}(G)\right|(1 \times 3)+\left|E_{3}(G)\right|(2 \times 2)+\left|E_{4}(G)\right|(2 \times 3)$

$=102 \times 2^{2 n-1}-81$.

3. ${ }^{m} M_{2}(G)=\sum_{u v \in E(G)} \frac{1}{d_{u} d_{v}}$

$=\left|E_{1}(G)\right| \frac{1}{1 \times 2}+\left|E_{2}(G)\right| \frac{1}{1 \times 3}+\left|E_{3}(G)\right| \frac{1}{2 \times 2}+\left|E_{4}(G)\right| \frac{1}{2 \times 3}$

$=11 \times 2^{2 n-1}-\frac{17}{4}$.

4. $R M_{2}(G)=\sum_{u v \in E(G)}\left(d_{u}-1\right)\left(d_{v}-1\right)$

$=\left|E_{1}(G)\right|(1-1)(2-1)+\left|E_{2}(G)\right|(1-1)(3-1)+\left|E_{3}(G)\right|(2-1)(2-1)$

$=27 \times 2^{2 n}-11$.

$$
+\left|E_{4}(G)\right|(2-1)(3-1)
$$

\subsection{Theorem 6}

Let $G$ be the schematic representation of thesecond-generation poly (amidoamine) dendrimer. Then the Randić index $R(G)$, the reciprocal Randić index $R R(G)$ and the reduced reciprocal Randić index $R R R(G)$ for $\mathrm{G}$ are
1. $R(G)=\left(\frac{3}{2 \sqrt{2}}+\frac{3}{\sqrt{3}}+\frac{9}{\sqrt{6}}+\frac{9}{2}\right) \times 22^{n}-\left(\frac{3}{\sqrt{3}}+\frac{6}{\sqrt{6}}+\frac{9}{2}\right)$.
2. $R R(G)=\left(\frac{3}{\sqrt{2}}+3 \sqrt{3}+9 \sqrt{6}+18\right) 2^{2 n}-(3 \sqrt{3}+6 \sqrt{6}+18)$.
3. $\operatorname{RRR}(G)=(9+9 \sqrt{2}) 2^{2 n}-(9+6 \sqrt{2})$.

\section{Proof.}

Using the edge partitions of the Schematic representation of the second-generation poly (amidoamine) dendrimer and by the formulas of different versions of the Randic indices, we get

$$
\begin{aligned}
\text { 1. } & R(G)=\sum_{u v \in E(G)} \frac{1}{\sqrt{d_{u} d_{v}}} \\
= & \left|E_{1}(G)\right| \frac{1}{\sqrt{1 \times 2}}+\left|E_{2}(G)\right| \frac{1}{\sqrt{1 \times 3}}+\left|E_{3}(G)\right| \frac{1}{\sqrt{2 \times 2}}+\left|E_{4}(G)\right| \frac{1}{\sqrt{2 \times 3}} \\
= & \left(\frac{3}{2 \sqrt{2}}+\frac{3}{\sqrt{3}}+\frac{9}{\sqrt{6}}+\frac{9}{2}\right) \times 22^{n}-\left(\frac{3}{\sqrt{3}}+\frac{6}{\sqrt{6}}+\frac{9}{2}\right) . \\
\text { 2. } & R R(G)=\sum_{u v \in E(G)} \sqrt{d_{u} d_{v}} \\
= & \left|E_{1}(G)\right| \sqrt{1 \times 2}+\left|E_{2}(G)\right| \sqrt{1 \times 3}+\left|E_{3}(G)\right| \sqrt{2 \times 2}+\left|E_{4}(G)\right| \sqrt{2 \times 3}
\end{aligned}
$$




$$
\begin{aligned}
& =\left(\frac{3}{\sqrt{2}}+3 \sqrt{3}+9 \sqrt{6}+18\right) 2^{2 n}-(3 \sqrt{3}+6 \sqrt{6}+18) . \\
& \text { 3. } \begin{aligned}
R R R(G)=\sum_{u v \in E(G)} \sqrt{\left(d_{u}-1\right)\left(d_{v}-1\right)} \\
=\left|E_{1}(G)\right| \sqrt{(1-1)(2-1)}+\left|E_{2}(G)\right| \sqrt{(1-1)(3-1)}+\left|E_{3}(G)\right| \sqrt{(2-1)(2-1)} \\
+\left|E_{4}(G)\right| \sqrt{(2-1)(3-1)}
\end{aligned} \\
& =(9+9 \sqrt{2}) 2^{2 n}-(9+6 \sqrt{2}) .
\end{aligned}
$$

\section{Conclusion}

In this work, we considered three types of dendrimers; polyacetal dendrimers with POSS core, the polyester dendrimers and polyamidoamine dendrimer which are used in drug delivery and Zagreb index and the Randic index were calculated using the edge set partitions to these dendrimers. These two indices are the mostly used vertex degree based topological indices in the field of drug design and developments. We also calculated the other versions of the Zagreb index such as the second Zagreb index, the second modified Zagreb index and the reduced second Zagreb index and the other versions of the Randic indices such as the reciprocal Randić index and the reduced reciprocal Randić index for these dendrimers. Closed formulas for all these indices were derived. A deep insight in the topological structure of these dendrimers could be found by these results.

\section{References}

1. M. Sherikhpour, L. Barani, and A. Kasaeian, J. Control. Rel. 253, 97 (2017). https://doi.org/10.1016/j.jconrel.2017.03.026

2. P. L. Brannon and J.O. Blanchette. Adv. Drug Delivery Rev. 56, 1649 (2004). https://doi.org/10.1016/j.addr.2004.02.014

3. H. -J. Zhang, X. Zhao, L. -J. Chen, C. -X. Yang, and X. -P. Yan, Talanta 121209 (2020). https://doi.org/10.1016/j.talanta.2020.121209

4. C. C. Lee, J. A. Mackay, J. M. Frechet, F. C. Szoka, Nat. Biotechnol. 23, 1517 (2005). https://doi.org/10.1038/nbt1171

5. S. Svenson and D. A. Tomalia, Adv. Drug Delivery Rev. 57, 2106 (2005). https://doi.org/10.1016/j.addr.2005.09.018

6. H. J. Hsu, J. Bungo, S. -R. Lee, and S. Hong, WIREs Nanomed. Nanobiotechnol. 9, e1409 (2017). https://doi.org/10.1002/wnan.1409

7. R. M. Kannan, E. Nance, S. Kannan, and D. A. Tomalia, J. Intern. Med. 276, 579 (2014). https://doi.org/10.1111/joim.12280

8. J. Hu, T. Xu, and Y. Cheng, Chem. Rev. 112, 3856 (2012). https://doi.org/10.1021/cr200333h

9. B. K. Nanjwade, H. M. Bechra, K. D. Ganesh, F. V Manvia, and N. K. Veerendra, Eur. J. Pharmaceut. Sci. 38, 185 (2009). https://doi.org/10.1016/j.ejps.2009.07.008

10. J. Manikkath, A. Mannikath, G. V. Shavi, K. Bhat, and S. Mutalik, J. Drug Delv. Sci. Technol. 41, 334 (2017). https://doi.org/10.1016/j.jddst.2017.07.021

11. H. J. Wiener, J. Amer. Chem. Soc. 69, 17 (1947). https://doi.org/10.1021/ja01193a005

12. J. C. Dearden, The Use of Topological Indices in QSAR and QSPR Modeling, in Advances in QSAR Modeling (Springer Publishers, 2017) pp. 57-88.

13. E. Estrada, G. Patlewicz, and E. Uriarte, Ind. J. Chem. 42, 1315 (2003).

14. R. Gozalbes, J. P. Doucet, and F. Derouin, Cur. Drug Targets - Infect. Disorders 2, 93 (2002). https://doi.org/10.2174/1568005024605909

15. T. P. Jude, E. Panchadcharam, and K. Masilamani, Ceylon J. Sci. 48, 4 (2019). http://doi.org/10.4038/cjs.v48i4.7677 
16. A. Aslam, Y. Bashir, S. Ahmad, and W. Gao, Zeitschrift Naturforschung A72 (2017). https://doi.org/10.1515/zna-2017-0081

17. S. M. Kang, M. A. Zahid, A. R. Virk, W. Nazeer, and W. Gao, Open Chem. 61 (2018).

18. I. Gutman and N. Trinaistic, Chem. Phys. Lett. 77, 535 (1972).

19. J. Hao. MATCH Commun. Math. Comput. Chem. 65, 659 (2011).

20. M. Randic, J. Am. Chem. Soc. 97, 6609 (1974). https://doi.org/10.1021/ja00856a001

21. X. Li and Y. Shi, MATCH Commun. Math. Comput. Chem. 59, 127 (2008).

22. O. Favaron, M. Maheo, J. F. Sacle, Discr. Math. 111, 197 (1993). https://doi.org/10.1016/0012$\underline{365 X(93) 90156-\mathrm{N}}$

23. I. Gutman, B. Furtula, and C. Elphic, MATCH Commun. Math. Comput. Chem. 72, 617 (2014).

24. D. Huang and W. Decheng, Mater. Sci. Eng. C 90, 713 (2018).

25. M. Liu and J. M. J. Fréchet, Res. Focus 2, 393 (1999). https://doi.org/10.1016/S1461$\underline{5347(99) 00203-5}$ 